\title{
Two Novel Heterozygote Mutations of ATM in a Chinese Family with Dystonia-Dominant Ataxia Telangiectasia and Literatures Review
}

Zhijun Liu ( $\square$ liuzhijunhubei@163.com )

Huazhong University of Science and Technology Tongji Medical College First Clinical College: Wuhan Union Hospital https://orcid.org/0000-0001-6252-9477

\section{Ming-Feng You}

Huazhong University of Science and Technology Tongji Medical College First Clinical College: Wuhan Union Hospital

\section{Ya-Ling Wang}

Huazhong University of Science and Technology Tongji Medical College First Clinical College: Wuhan Union Hospital

\section{Yan Xu}

Huazhong University of Science and Technology Tongji Medical College First Clinical College: Wuhan Union Hospital

\section{Research Article}

Keywords: Ataxia-telangiectasia, targeted exome-sequencing, ATM, dystonia, Chinese

Posted Date: December 21st, 2021

DOI: https://doi.org/10.21203/rs.3.rs-27193/v2

License: (c) (i) This work is licensed under a Creative Commons Attribution 4.0 International License. Read Full License 


\section{Abstract}

Ataxia-telangiectasia (A-T) is an autosomal recessive disorder with high clinical heterogeneity. A-T may present in complicated variable forms, mainly including classic $A-T$ and milder forms. Contrary to the classic A-T, the milder form does not present the cardinal features of A-T, including ataxia and telangiectasia. A few ATM mutations have been reported in variant A-T cases manifested as isolated dystonia without any signs of classical A-T. To date, more than 400 disease-related ATM mutations have been identified in patients with A-T. In this study, target exome-sequencing was performed in an AT pedigree with predominant dystonia. Two novel ATM mutations, p.I2683T and p.S2860P, were identified in the family. We then reviewed previously published literatures of genetically confirmed A-T cases with predominant dystonia and summarized the clinical characteristics of dystonia-dominant A-T. To our knowledge, this is the first report of A-T patient with predominant dystonia in China. Dystonia may appear as one of the predominant manifestations or initial symptom of A-T. ATM genetic testing should be early considered for those patients with predominant dystonia, despite without accompanying ataxia or telangiectasia.

\section{Introduction}

Ataxia-telangiectasia (A-T) is a rare autosomal recessive disorder characterized by progressive cerebellar degeneration, telangiectasia, immunodeficiency, radiosensitivity, recurrent respiratory tract infections, and increased incidence of cancer[1]. Most people with A-T developed the disease in early childhood and die from malignancies or respiratory failure in the second or third decade of life[2].

A-T may present in complicated variable forms, mainly including classic A-T which is caused by truncating ataxia telangiectasia mutated (ATM) mutations in both alleles leading to total loss of ATM kinase activity and milder forms (also known as variant A-T) often associated with missense or leaky splice mutations[3]. Patients with classic A-T usually develop an ataxic gait in their early childhood and become wheelchair dependency by adolescence. Contrary to the classic A-T, the milder form does not present the cardinal features of A-T, such as ataxia, telangiectasia, and immunodeficiency. Other characteristics of movement disorders including chorea, myoclonic jerks, resting tremor, and dystonia are now well recognized as the presenting manifestations of variant A-T. Recently, a systematic review of movement disorder phenomenology and systemic features in A-T patients found that $89 \%(186 / 210)$ cases manifested dystonia and $18 \%(24 / 130)$ presented dystonia as the initial manifestations of A-T[4]. Notably, a few ATM mutations have been reported in variant A-T cases manifested as isolated generalized or segmental dystonia without any signs of classical A-T.

A-T is caused by mutations in the ATM gene which plays a critical role in regulating DNA double-strand breaks (DSBs) repairs pathway. To date, more than 400 disease-related ATM mutations have been identified in patients with A-T. Among the ATM mutations, the majority (about $70 \%$ ) of variants are truncating changes resulting in premature protein termination[2]. 
In this study, we reported a Chinese A-T family with two novel compound heterozygote mutations in the ATM gene. To our knowledge, this is the first report of A-T patient with predominant dystonia in China. Our findings broaden the molecular and clinical spectrum of Chinese A-T patients.

\section{Methods}

\subsection{Participants}

The pedigree was collected from the Neurology Department of Wuhan Union hospital. DNA samples were obtained from all the participants, including the proband, his affected older sister and their parents. Magnetic resonance imaging (MRI) of brain and cervical, ultrasound of heart and abdominal, electroencephalogram (EEG), and blood biochemical analysis (include detection of a-fetoprotein (AFP), immunoglobulin(Ig), and ceruloplasmin) were conducted. An additional 200 normal individuals without any neurological disorders were included as controls. Written informed consents were obtained from all participants. This study was approved by the ethics committee of Wuhan Union Hospital.

\subsection{Targeted exome-sequencing}

Genomic DNA was extracted from peripheral blood samples by DNA extraction Kit (Qiagen, Germany). A panel was designed to cover 101 genes associated with movement disorders (Supplementary table 1). Deep sequencing was performed using Illumina Hiseq2000 system (GrandOmics Biosciences Co, China). Sequencing analysis were performed as we described previously[5]. In order to screen for large deletions or duplications of Dopa-responsive dystonia (DRD) associated genes ( $G C H 1, T H$, and $S G C E$ ), the Multiplex ligation-dependent probe amplification assay (MLPA) analysis were performed by reference to the method previously reported[6].

\subsection{Sanger sequencing}

Sanger sequencing was used to validate the candidate variants after data analysis. Forward and reverse primers were designed to amplify the fragments covering the variant sites. The fragments were sequenced on $A B I 3530 x L$ DNA Sequencer. Co-segregation analysis was conducted through screening for the confirmed variants in the proband's older sister and parents.

\section{Results}

\subsection{Results of genetic testing}

No large deletions or duplications of $G C H 1, T H$, and SGCE, were found in the proband. About $94.5 \%$ of the target bases were covered with at least 50X, and the mean depth of coverage for all target regions was 170. After filtering, two novel ATM variants, c.T8048C(p.I2683T) and c.T8578C(p.S2860P), with probable pathogenicity were identified in the proband. The two variants were confirmed in the proband and his affected older sister by Sanger sequencing. The two variants were found heterozygous in their unaffected parents. These two variants were absent in all public variant databases (1000G frequency, ESP6500, and 
ExAC frequency) and our 200 normal individuals. All two variants were predicted as harmful effects by the SIFT, PolyPhen-2, and MutationTaster software. According to the ACMG standards and guidelines, the two variants were classified as likely pathogenic variants.

\subsection{Clinical characteristics}

The proband (II:2), a 15-year-old boy, was delivered by forceps with no birth complications and had a normal early milestones. He started walking around one year of age and had normal speech. He presented at our clinic complaining of neck pain and stiffness since 9 years old, which progressively worsened over the period of 2-3 years. He developed mild involuntary head shake at age 12 . Then he experienced episodes of involuntary turning of the head to the left and cervical hyperextension, postural hand tremor, and blepharospasm at age 13 . On examination at age 15 , he had severe dystonia with dominant craniocervical involvement. The jerky right torticollis, postural hand tremor, and choreiform facial movements were present. Eye movements were normal with normal saccades responses and pursuit eye movements. He had normal muscle power and reflexes, and a slightly increased muscle tone in left upper limb. Sensory examination was unremarkable. There were no cerebellar signs. His cognitive function remains intact, with a Mini-Mental State Exam (MMSE) and Montreal Cognitive Assessment (MoCA) score of 28/30 and 25/30, respectively. Eye ophthalmic examination was unremarkable.

His older sister(Il:1) was a 19-year-old girl and experienced similar symptoms since age 12 years. She noted her first symptom of mild involuntary head shake while taking a photo at the age of 12 years. As the disease progresses, she gradually developed jerky right-sided torticollis, postural hand tremor, oromandibular dystonia. She had a recurrence episode of transient amnesia, which occurred 2-3 times per year. Her examination was further marked by severe cervical and oromandibular dystonia, a slightly increased muscle tone in left upper limb, and postural hand tremor. The deep tendon reflexes were all absent, with flexor plantar responses. She showed normal saccades and pursuit eye movements. Her cognitive function was normal.

No known consanguinity was noted in the family. The proband's grandmother had a gastric cancer and died at age 50. The detailed clinical results are summarized in Table 1. The serum AFP levels were significantly increased in all of the two patients, whereas serum levels of $\operatorname{lgG}, \lg \mathrm{A}, \mathrm{C} 3$, and $\mathrm{C} 4$ was decreased. Other laboratory tests including cholesterol, creatinine alkaline, lactate dehydrogenase (LDH), and ceruloplasmin were normal. The brain and spinal cord MRI, ultrasound of heart and abdominal, and electroencephalogram (EEG) were unremarkable.

\section{Discussion}

In this study, we presented a clinical genetic study of Chinese A-T family presenting with atypically craniocervical dystonia through targeted exome-sequencing and further reviewed previously published 
dystonia-dominant A-T cases. No signs of ocular motor apraxia, cerebellar ataxia, as well as telangiectasia were observed in present case.

Dystonia occurring either in isolation or in combination with other clinical features, has been described in variant A-T patients. Dystonia affecting the neck, head, trunk and limbs, may be present as initial symptoms in about $18 \%(24 / 130)$ of A-T patients[4]. The present case showed obviously isolated segmental dystonia without any signs of ataxia and telangiectasias. After reviewed previously published literatures of genetically confirmed A-T cases with predominant dystonia, we found that these dystoniadominant A-T cases may have a significantly different clinical picture compared to the typical A-T. In total, 11 pedigrees including 29 patients (13 Female, 16 Male patients) with ATM mutations were reviewed[7-14] (Table 1). The median age of onset was 10.67 years, ranging from 1 to 20 years old. The group of dystonia-dominant A-T cases showed isolated dystonic features or accompanied by choreoathetosis, tremor and/or myoclonic jerks, but without involvement of ataxia and telangiectasias. Other phenotypes, including peripheral neuropathy, delayed motor development, speech and swallowing difficulties, could also be seen in the group. A few patients also experienced recurrent pneumonia and increase risk of malignancy. Overall, the patients with dystonia-dominant A-T tend to have a later-onset and slower progression of the disease. However, the relationship between dystonic phenotype and particular ATM genotype in variant A-T is not well understood. As previously reported, mutations in exon 42 of the ATM gene may predispose to dystonia-dominant A-T, which is needed to be confirmed in further analysis[15]. Notably, the transient amnesia observed in present case was rare and first described in patients with A-T. Further investigation and analysis of amnesia presentation in patients with A-T are needed.

In line with previous study, significantly elevated AFP levels and decreased serum levels of immunoglobulin and complements (IgG, IgA, C3 and C4) were observed in this family. Previous studies revealed that elevated IgM levels are indicative of a distinct and more severe phenotype[16]. IgM levels was normal in present case, which suggests the mild phenotype observed in our case. Unfortunately, the ATM kinase activity was not detection in our patients.

The neuropathological hallmark of A-T is cerebellar Purkinje cell loss, leading to cerebellar symptoms and degeneration[17]. The exact pathogenesis of dystonia in A-T remains less clear. A review of pathological findings in 17 patients with A-T and movement disorders found that neurona loss occurs in basal ganglia and brain stem nuclei[18]. Previous positron emission tomography (PET) study revealed reduced glucose metabolism in the cerebellum of A-T patients and increased metabolism in the globus pallidus which often resulted in decreased motor performance[19]. Additional studies are required to assess the association of dystonia with cerebellum and basal ganglia degeneration in A-T patients.

In conclusion, our study expanded the clinical and molecular spectrum of dystonia-dominant A-T in China. Dystonia may appear as one of the predominant manifestations or initial symptom of A-T. Isolated dystonia without any signs of ataxia or telangiectasias may result in the delays in the diagnosis of A-T. 
ATM genetic testing should be early considered for those patients with predominant dystonia, despite without accompanying ataxia or telangiectasia.

\section{Declarations}

\section{Acknowledgements}

We would like to thank all the subjects for their help and active participation in this study. We thank the reviewers for the comments and suggestions.

\section{Author contributions}

Dr. Liu performed the molecular genetic analysis and participated in its design and drafted the manuscript. Dr. You and Wang collected data from all participants and performed molecular genetic analysis. Prof. Xu conceived of the study, and participated in its design and helped to draft the manuscript. All authors read and approved the final manuscript.

\section{Funding}

This work was supported by the grant from the National Natural Science Foundation of China (No 81873734).

\section{Data Availability}

All data generated or analyzed during this study are included in this published article and its supplementary information files.

\section{Declaration of competing interest}

The authors report no potential conflicts of interest.

\section{Ethics declarations}

This study was approved by the ethics committee of Wuhan Union Hospital. Written informed consents were obtained from all participants.

\section{References}


1. Rothblum-Oviatt C, Wright J, Lefton-Greif MA, McGrath-Morrow SA, Crawford TO, Lederman HM (2016) Ataxia telangiectasia: a review. Orphanet J Rare Dis 11(1):159

2. Micol R, Ben Slama L, Suarez F, Le Mignot L, Beaute J, Mahlaoui N, Dubois d'Enghien C, Lauge A, Hall J, Couturier J, Vallee L, Delobel B, Rivier F, Nguyen K, Billette T, de Villemeur JL, Stephan P, Bordigoni Y, Bertrand N, Aladjidi JM, Pedespan C, Thomas I, Pellier M, Koenig O, Hermine C, Picard D, Moshous B, Neven F, Lanternier S, Blanche M, Tardieu M, Debre A, Fischer D, Stoppa-Lyonnet CN, Investigators (2011) Morbidity and mortality from ataxia-telangiectasia are associated with ATM genotype. J Allergy Clin Immunol 128(2):382-9e1

3. Taylor AM, Lam Z, Last JI, Byrd PJ (2015) Ataxia telangiectasia: more variation at clinical and cellular levels. Clin Genet 87(3):199-208

4. Levy A, Lang AE (2018) Ataxia-telangiectasia: A review of movement disorders, clinical features, and genotype correlations. Mov Disord 33(8):1238-1247

5. Liu ZJ, Lin HX, Wei Q, Zhang QJ, Chen CX, Tao QQ, Liu GL, Ni W, Gitler AD, Li HF, Wu ZY (2019) Genetic Spectrum and Variability in Chinese Patients with Amyotrophic Lateral Sclerosis. Aging Dis 10(6):1199-1206

6. Sun ZF, Zhang YH, Guo JF, Sun QY, Mei JP, Zhou HL, Guan LP, Tian JY, Hu ZM, Li JD, Xia K, Yan XX, Tang BS (2014) Genetic diagnosis of two dopa-responsive dystonia families by exome sequencing. PLOS ONE 9(9):e106388

7. Simonin C, Devos D, Vuillaume I, de Martinville B, Sablonniere B, Destee A, Stoppa-Lyonnet D, Defebvre $L$ (2008) Attenuated presentation of ataxia-telangiectasia with familial cancer history. $J$ Neurol 255(8):1261-3

8. Carrillo F, Schneider SA, Taylor AM, Srinivasan V, Kapoor R, Bhatia KP (2009) Prominent oromandibular dystonia and pharyngeal telangiectasia in atypical ataxia telangiectasia. Cerebellum $8(1): 22-27$

9. Saunders-Pullman R, Raymond D, Stoessl AJ, Hobson D, Nakamura K, Pullman S, Lefton D, Okun MS, Uitti R, Sachdev R, Stanley K, San Luciano M, Hagenah J, Gatti R, Ozelius LJ, Bressman SB (2012) Variant ataxia-telangiectasia presenting as primary-appearing dystonia in Canadian Mennonites. Neurology 78(9):649-657

10. Meissner WG, Fernet M, Couturier J, Hall J, Lauge A, Henry P, Stoppa-Lyonnet D, Tison F (2013) Isolated generalized dystonia in biallelic missense mutations of the ATM gene. Mov Disord 28(13):1897-9

11. Cummins G, Jawad T, Taylor M, Lynch T (2013) Myoclonic head jerks and extensor axial dystonia in the variant form of ataxia telangiectasia. Parkinsonism Relat Disord 19(12):1173-4

12. Kuhm C, Gallenmuller C, Dork T, Menzel M, Biskup S, Klopstock T (2015) Novel ATM mutation in a German patient presenting as generalized dystonia without classical signs of ataxia-telangiectasia. J Neurol 262(3):768-770

13. Lohmann E, Kruger S, Hauser AK, Hanagasi H, Guven G, Erginel-Unaltuna N, Biskup S, Gasser T (2015) Clinical variability in ataxia-telangiectasia. J Neurol 262(7):1724-7 
14. Necpal J, Zech M, Skorvanek M, Havrankova P, Fecikova A, Winkelmann J, Jech R (2018) Ataxia Telangiectasia Gene Mutation in Isolated Segmental Dystonia Without Ataxia and Telangiectasia. Mov Disord Clin Pract 5(1):89-91

15. Charlesworth G, Mohire MD, Schneider SA, Stamelou M, Wood NW, Bhatia KP (2013) Ataxia telangiectasia presenting as dopa-responsive cervical dystonia. Neurology 81(13):1148-1151

16. Krauthammer A, Lahad A, Goldberg L, Sarouk I, Weiss B, Somech R, Soudack M, Pessach IM (2018) Elevated IgM levels as a marker for a unique phenotype in patients with Ataxia telangiectasia. BMC Pediatr 18(1):185

17. Verhagen MM, Martin JJ, van Deuren M, Ceuterick-de Groote C, Weemaes CM, Kremer BH, Taylor MA, Willemsen MA, Lammens M (2012) Neuropathology in classical and variant ataxia-telangiectasia. Neuropathology: official journal of the Japanese Society of Neuropathology 32(3):234-244

18. Koepp M, Schelosky L, Cordes I, Cordes M, Poewe W (1994) Dystonia in ataxia telangiectasia: report of a case with putaminal lesions and decreased striatal [123l]iodobenzamide binding. Mov Disord 9(4):455-9

19. Volkow ND, Tomasi D, Wang GJ, Studentsova Y, Margus B, Crawford TO (2014) Brain glucose metabolism in adults with ataxia-telangiectasia and their asymptomatic relatives, Brain: a journal of neurology 137(Pt 6) 1753-61

\section{Tables}

Due to technical limitations, table 1 is only available as a download in the Supplemental Files section.

\section{Figures}




\section{A}

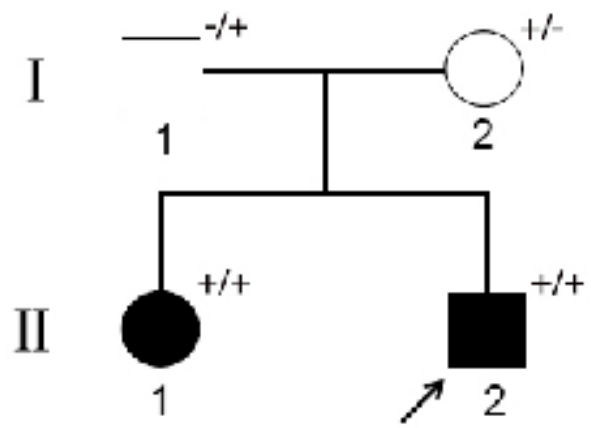

Family

B
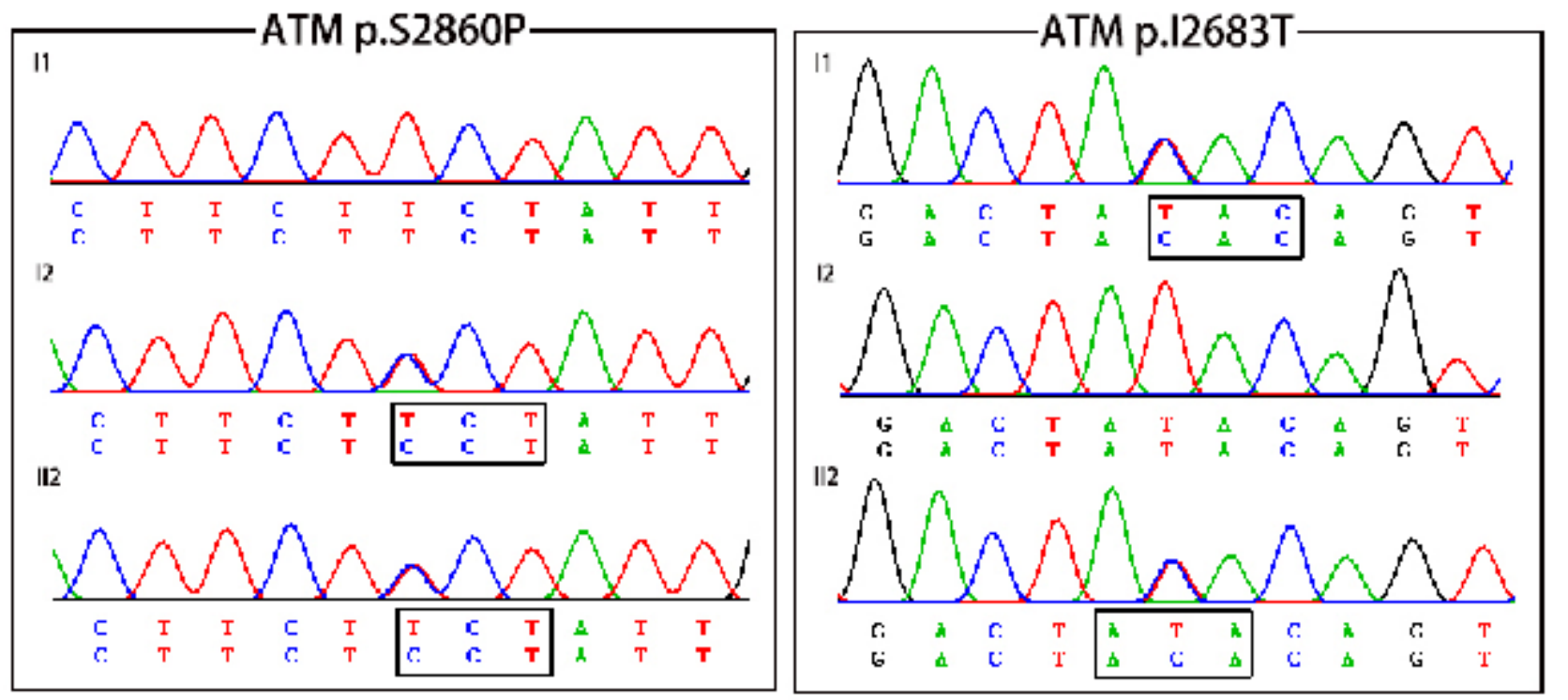

\section{Figure 1}

A. The pedigree of A-T patients in present study. Squares indicate males; circles indicate females; filled symbols indicate affected individuals; arrows indicate the probands; "+/+" indicate two mutant alleles; "+/-" or "-/+" indicate mutation occurring in one of two alleles. B. Chromatograms of the proband and the proband's parents.

\section{Supplementary Files}

This is a list of supplementary files associated with this preprint. Click to download.

- Table1.docx

- Supplementarytable1..xlsx 